\title{
DEVELOPMENT AND EXPERIMENTAL VALIDATION OF A CORRELATION MONITOR TOOL BASED ON THE ENDOGENOUS PULSED NEUTRON SOURCE TECHNIQUE
}

\author{
Mohammad Arkani ${ }^{1)}$, Hossein Khalafi ${ }^{1)}$, Naser Vosoughi ${ }^{2)}$, Samad Khakshournia ${ }^{1)}$ \\ 1) Reactor and Nuclear Safety Research School, Nuclear Science \& Technology Research Institute (NSTRI), 143995-1113, Tehran, Iran \\ \markani@aeoi.org.ir,+982188221103,hkhalafi@aeoi.org.ir,skhakshournia@aeoi.org.ir) \\ 2) Sharif University of Technology, Azadi Avenue, 11365-11155, Tehran, Iran (nvosoughi@sharif.ir)
}

\begin{abstract}
A correlation measuring tool for an endogenous pulsed neutron source experiment is developed in this work. Paroxysmal pulses generated by a bursts of neutron chains are detected by a 10-kbit embedded shift register with a time resolution of $100 \mathrm{~ns}$. The system is implemented on a single reprogrammable device making it a compact, cost-effective instrument, easily adaptable for any case study. The system was verified experimentally in the Esfahan heavy-water zero power reactor (EHWZPR). The results obtained by the measuring tool are validated by the Feynman- $\alpha$ experiment, and a good agreement is seen within the boundaries of statistical uncertainties. The theory of the methods is briefly initiated in the text. Also, the system structure is described, the experimental results and their uncertainties are discussed, and neutron statistics in EHWZPR is examined experimentally.
\end{abstract}

Keywords: endogenous pulsed neutron source experiment, neutron noise fluctuation, zero power reactor noise, Esfahan heavy-water zero power reactor, neutron correlation.

(C) 2017 Polish Academy of Sciences. All rights reserved

\section{Introduction}

Neutron noise fluctuations observed in a form of correlated random pulses or signals from neutron detectors inside or nearby a nuclear reactor core are an object of a well-established and standard branch of studies, applications, and diagnosis in the field of stochastic experimental nuclear reactor physics. The knowledge of data treatment of neutron noise fluctuations originated by microscopic characteristics of the core is called zero power reactor noise (ZPRN) theory. The ultimate aim of ZPRN analysis is to measure and diagnose the kinetic parameters of the reactor core which are determined by the geometry and material composition of the system [1]. The main advantageous feature of the method is its complete independence from external excitation or perturbation of the system itself which makes it a valuable technique. The experimental methods of ZPRN analysis are categorized by several different techniques that might be classified in five major categories: analysis of probability profiles of neutron counts, analysis of moments of neutron count distributions, analysis of correlation among neutron counts in the time domain, correlation polarities in the time domain, and analysis of reactor power level in the frequency domain [2-6]. Each technique has its own data analysis and experimental data collection method [7-18]. The endogenous (inherent) pulsed neutron source technique is a branch of correlation analysis of neutron counts in the time domain [3] (the third item in the above categories of ZPRN analysis). The basic principle of the technique is the conditional start of a multichannel scaler. Bursts of neutron chains are detected by a conditional pulse counter and the delay time-dependent correlation function of the correlative chain neutrons is registered by the multichannel scaler. The time at which a burst of neutron chains 
is detected is the time origin for all recorded data. A prompt neutron decay constant of the core is determined by analysing the acquired data.

Neutrons released by fission chains are correlated in time. Two types of correlations are possible. A positive correlation - the existence of a pulse or event at a certain time makes the probability of another pulse or event increased immediately afterwards; this kind of correlation is seen in nuclear reactor systems where neutrons are linked in time by fission chains. A negative correlation - the existence of a pulse or an event at a certain time makes the probability of another pulse or event decreased immediately afterwards; this type of correlation is usually generated by the dead time behaviour of particle detection systems. Radioactive particles emitted by decaying type radiation sources are random sequences obeying the Poisson distribution law. The time interval between consecutive pulses is not invariant but follows a purely exponential probability. This kind of random sequences is usually generated by constant sources of decaying types, whose half-lives are practically infinite in comparison with the observation time. A random sequence is not a correlated one; every decaying process is an independent nuclear process $[19,20]$.

Considering the statistics of the above mentioned random processes, three different values of variance to mean (VTM) ratio of the counts are possible: $V T M>1$ for a positive correlation (in a multiplying medium), $V T M=1$ for a pure Poisson process, and $V T M<1$ for a process of negative correlation. Note that $V T M>1$ means that the neutron fluctuations inside the medium are larger than those in the corresponding pure Poisson process (a Poisson process with the same mean value of neutron counts). It is noticeable that a cluster of condensed pulses appearing in time due to a burst of neutron chains means a long time separation of the bursts by the background or random statistics of the system.

In a multiplying medium, such as a subcritical system, random processes are not purely correlated in time. The whole process is the superimposition of an uncorrelated random process and the correlated process of neutrons originated from common ancestors of fission chains. The ratio of the correlated and uncorrelated portions of the process is usually called a signal to background ratio (SBR) which is a measure of correlation in the system. In fact, the uncorrelated portion is much larger than the correlated one, and the extraction of data needs a wisely adjusted experimental set-up based on physical knowledge about system statistics and its properties. Since the Esfahan heavy-water zero power reactor (EHWZPR), the case study of the problem in the present work, is a heavy-water reactor, producing photoneutrons is probable. Therefore, the background portion of the neutron statistics is physically strong in comparison with the correlated sequences in the system.

Some physical simplifications assumed in this work are summarized as follows:

- The first fundamental assumption for ZPRN analysis techniques is an ergodic hypothesis; the reactor is in steady-state operating conditions, and the evolution of neutron population is expected to be stationary. The reactor to be studied is stable, and its stochastic features do not vary with time. If different measurements are repeated in different periods, the same results within the statistical uncertainties are seen.

- The basic assumption of the background theory of the experiments is the point reactor supposition. Therefore, the point reactor assumption should be satisfied by the conditions of the problem. The results obtained by a detector positioned in the core should represent the features of the whole system.

- The delayed neutrons and photoneutrons are considered as the background radiation in EHWZPR. Only prompt neutron fluctuations are analysed.

Correlation monitors have been used in different applications for measurement of physical parameters [21, 22]. In reactor noise studies, the theory has been studied lately, and different apparatuses have been developed for ZPRN experiments [3-4, 9]. Analogue monitors based 
on a single rate meter or two inter-compensated rate meters and digital monitors are also applied [16-17, 23].

The main idea of the endogenous pulsed neutron source method is to compare the neutron counts with the average or a threshold level. Bursts of neutron chains are detected and selected by a comparator, then, a recording apparatus is triggered to collect the correlation data. This process is repeated for many times until acceptable statistical accuracy is met. In the present work, a correlation monitor is developed based on field programmable gate arrays (FPGAs). A shift register as a proven technique of neutron correlation measurement [25-25] is employed. A 10-kbit serial-in-serial-out shift register is implemented in the design to record enough history of the random input process with a fine discretization of time. Parameters of the design are possible to be adjusted by the experimenter according to the case study of the problem. Considering the physical properties of EHWZPR $\left(\alpha_{c} \approx 6.84 \pm 0.088 \mathrm{~s}^{-1}\right.$, the prompt neutron decay constant at critical state of the core) [13], one millisecond of the input sequence is stored in the shift register at $100 \mathrm{~ns}$ of time resolution. Also, an embedded zero advanced time multichannel scaler is designed and built into the device. The neutron counts are registered by the device every millisecond after triggering by a digital burst detection circuit. The proposed equipment can save the accumulated data at a different number of scans performed on the burst of neutron chains. The system is tested and validated in EHWZPR by experiment, and the results are compared with the information obtained by the Feynman- $\alpha$ experiment. A good agreement between the results is seen. The proposed system is a cost-effective and compact instrument based on the state-of-the-art digital electronic technology. The data on neutron noise fluctuations in heavy-water moderated critical assemblies is published elsewhere [26] but regarding the characteristic neutron noise in heavy-water and graphite-reflected reactors - few papers have been published in the literature on the subject. Thus, the ZPRN analysis is mainly applied to the fast and light-water moderated systems [27-30]. Using the proposed measuring tool, an experimental study on neutron noise fluctuations in EHWZPR is performed. Also, the uncertainty of the results is analysed. The results obtained by the endogenous pulsed neutron source method are validated by the Feynman- $\alpha$ experiment.

\section{Background theory}

\subsection{Endogenous pulsed neutron source method}

The natural fluctuations of neutron density in a multiplying medium produce prompt paroxysmal pulses (due to bursts of neutron chains) which are higher than the average level, and they decay to lower levels according to the exponential law. The slope of the exponent is the prompt neutron decay constant of the core. The paroxysmal pulses are detected to generate the triggering signal. This detection leads to selecting the most important portion of the random sequence which contains information about correlated neutrons. As pulses are produced by the natural fluctuations, the method is named as endogenous or inherent pulsed neutron source experiment. Note that, in the pulsed neutron source experiment [31], bursts of neutron chains are induced by an external pulse mode neutron source. The equation describing the experimental results of the two methods are the same as:

$$
n(t)=C_{C} \exp (-\alpha t)+U_{C}
$$

where: $U_{C}$ is the mean neutron count (the background or uncorrelated count); $C_{C}$ is the neutron count of the starting channel (the correlated count); $\alpha$ is the prompt neutron decay constant, and $t$ is a time after detection of paroxysmal pulses (or after pulse injection in the pulsed neutron source method). Parameters of this equation are estimated by curve fitting to the experimental data. The mean neutron count in a time interval $T$ is defined as [3]: 


$$
U_{C}=\left\{\begin{array}{cl}
<F>\varepsilon T, & \text { in critical reactor } \\
\frac{S_{o} \varepsilon T}{\bar{v}\left(1-k_{\text {eff }}\right)}, & \text { in subcritical reactor drived by an external neutron source }
\end{array}\right.
$$

where: $\langle F\rangle=\frac{1}{T} \int_{0}^{T} F(t) d t$. Mean fission rate: $\varepsilon-$ neutron detection efficiency (number of neutrons detected per fission); $S_{o}$ - external neutron source power; $\bar{v}$ - Mean number of neutrons released per fission; $k_{\text {eff }}$ - effective multiplication factor.

Usually, the measuring time of the endogenous pulsed neutron source method is relatively long. The relation between the data collection time, $T_{\text {Collection, }}$ correlated counts, $C_{C}$, and other physical parameters of the system is introduced as (3) [7]. Note that $T_{\text {Collection }}$ is the amount of time which is taken by the experiment for data collection to meet an acceptable experimental uncertainty. It is clear that the data acquisition system starts by paroxysmal pulses and then stops when the scan is finished:

$$
T_{\text {Collection }}=\frac{C_{C}}{<F>\times \varepsilon \times T \times \theta},
$$

where $\theta$ is the product of a repetition rate and a signal to background ratio, which is defined by (4):

$$
\theta=R \times S B R,
$$

where: $S B R$ is a signal to background ratio; and $R$ is a repetition rate of paroxysmal neutron pulses. As the repetition rate increases, the number of scans per unit time is also increased. Therefore, the data collection time is decreased. A larger SBR means a lower background in comparison with the signal. A better signal to background ratio leads to a shorter time for data collection. As a result, there is an inverse relationship between $T_{\text {Collection }}$ and $\theta$. The $R$ and $S B R$ parameters are defined by (5) and (6), respectively:

$$
\begin{aligned}
& S B R=\frac{C_{C}}{U_{C}}, \\
& R=\frac{1}{T} \times \sum_{i=\gamma}^{\infty} P_{i},
\end{aligned}
$$

where: $P_{i}$ is the probability of counting $i$ pulses within the time interval $T$; and $\gamma$ is a threshold condition for detection of the bursts of neutron chains. Other parameters have the same definitions as given in the previous equations.

This type of technique can be considered as an intermediate method between the Rossi- $\alpha$ experiment [2] and the classic pulsed neutron source technique, showing similarities to both of them in the expression describing the experimental results (refer to (1)). Therefore, the endogenous pulsed neutron source technique enables to analyse neutron noise in thermal reactors for a reasonably acceptable measurement time, while the Rossi- $\alpha$ method is widely known as a time-consuming method in such systems. The endogenous pulsed neutron source experiment also features practical advantages when compared with pulsed neutron source measurements since the expensive apparatus of the pulsed neutron source is no longer necessary. Furthermore, the setting up of the experiment and the reactor operation procedure are considerably simplified. 


\subsection{Feynman- $\alpha$ method}

Neutrons in a non-multiplying medium generated by a Poisson neutron source have zero correlation with each other. Therefore, the random process observed at the output of the neutron detector is mostly similar to a Poisson distribution, the only distortion on the process is the dead time effect of the detection system [32]. Conversely, in a multiplying medium, neutrons are chain-correlated. Therefore, the process is different from a Poisson distribution and a larger standard deviation in comparison with a non-multiplying medium is seen. A larger value of standard deviation means larger fluctuations of neutron density in the core. A ratio of variance and mean of neutron counts in a specific time window $(\tau)$ is a measure of statistical fluctuations. It is defined as:

$$
V T M=\frac{\overline{C^{2}}-\bar{C}^{2}}{\bar{C}} .
$$

where: $C$ is the number of neutrons counted in a time window. As neutron chains in a nuclear reactor core are correlated in time, the distribution differs from the Poisson process. Therefore, besides uncorrelated neutron counts with a Poisson distribution (the background term or uncorrelated neutrons from other sources or other uncorrelated neutron chains), there also exist correlated neutrons. That is why the statistics deviate from the Poisson distribution. The degree of deviation is expressed by $Y$ parameter in the Feynman- $\alpha$ formula as [2]:

$$
\frac{\overline{C^{2}}-\bar{C}^{2}}{\bar{C}}=1+Y \text {, }
$$

the $Y$ parameter is expressed by (9):

$$
Y=\overline{\frac{v_{p}\left(v_{p}-1\right)}{\tau_{f}^{2}}} \frac{\varepsilon}{\alpha^{2}}\left(1-\frac{1-\exp (-\alpha \tau)}{\alpha \tau}\right),
$$

where: $\varepsilon$ is a neutron detection efficiency (the number of neutrons detected per fission); $\tau_{f}$ is the inverse of $v \Sigma_{\mathrm{f}}$ (the product of neutron velocity and microscopic fission cross-section), and $v_{p}$ is the average number of prompt neutrons released per fission. $Y$ is a measure of deviation from the Poisson distribution. $Y$ is determined at different values of time windows. The curve-fitting method is used to fit (9) to the experimental data to obtain an estimation of $\alpha$ parameter. A novel method of data analysis is proposed by [33] which reduces the machine time required for the experiment and increases the statistical accuracy. This technique is called bunching method. More details on the Feynman- $\alpha$ experiment are available elsewhere [3-4, 5-6, 34].

\section{System design and its architecture}

Neutron fluctuations in a multiplying medium are superimposition of correlated and uncorrelated random processes. Therefore, selected portions of the pulse sequences have to be recorded. As correlated pulse sequences are the desired data to be recorded, the trigger signal is asserted by positive fluctuations (burstsof neutron chains) to enable the data storage by the multichannel scaler (MCS). The heart of correlation monitor developed in the present work is a circuit for detecting bursts of neutron chains. Fig. 1 shows the method employed in this work for trigger generation. The first stage is a synchronizing block, for synchronization of random input pulses with a $10 \mathrm{MHz}$ system clock. A 10-kbit serial-in serial-out shift register provides a moving storage for the previous pulses during the last millisecond with a $100 \mathrm{~ns}$ time resolution. Regarding the dead time of the neutron detectors (a few microseconds) and the counting rate of the detector during the experiment ( $\sim 30000 \mathrm{cps})$, the time resolution is good enough. However, finer time discretization is also possible at the expense of additional hardware resource in the form of a deeper shift register. Input pulses and output pulses of the shift register block are counted by two separate 64-bit up-counters. By subtraction of the counts 
(counted by the two counter blocks), the number of pulses within the last millisecond of time is calculated. The result is compared with a pre-set threshold value for triggering the MCS block. In Fig. 2, a general schematic diagram of the system is presented. Except for the starting channel of the MCS from which its data are supplied by the circuit shown in Fig. 1, the upcounter block provides neutron counts in every millisecond after triggering. Any trigger signal starts the recording process by Nios II CPU interruption [35]. Communication with the computer as well as internal settings of the system are accomplished by the soft core processing unit. More details are shown in Figs. 1 and 2.

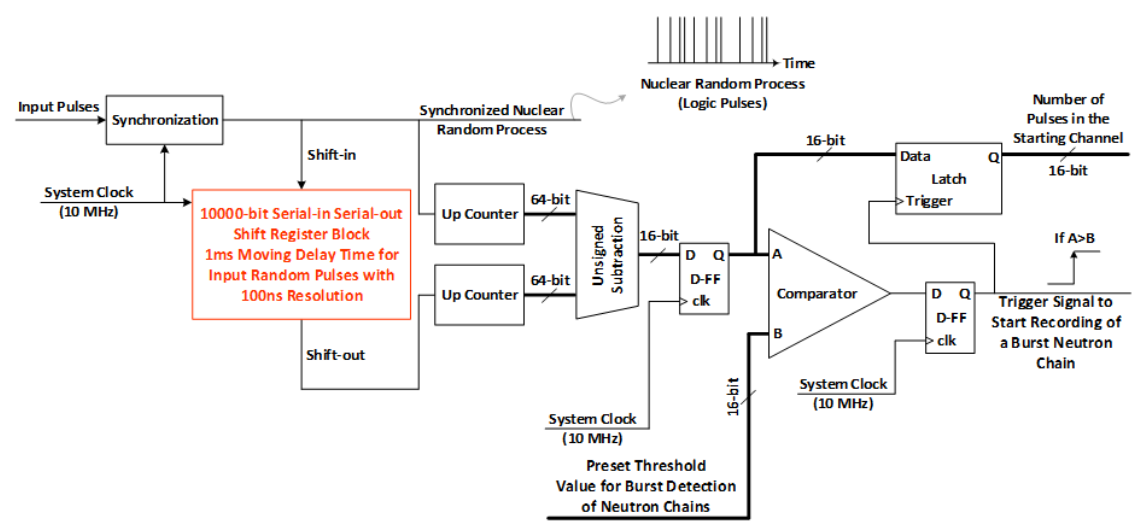

Fig. 1. The digital circuit for detection of positive fluctuations of neutron noise, generated by bursts of neutron chains.

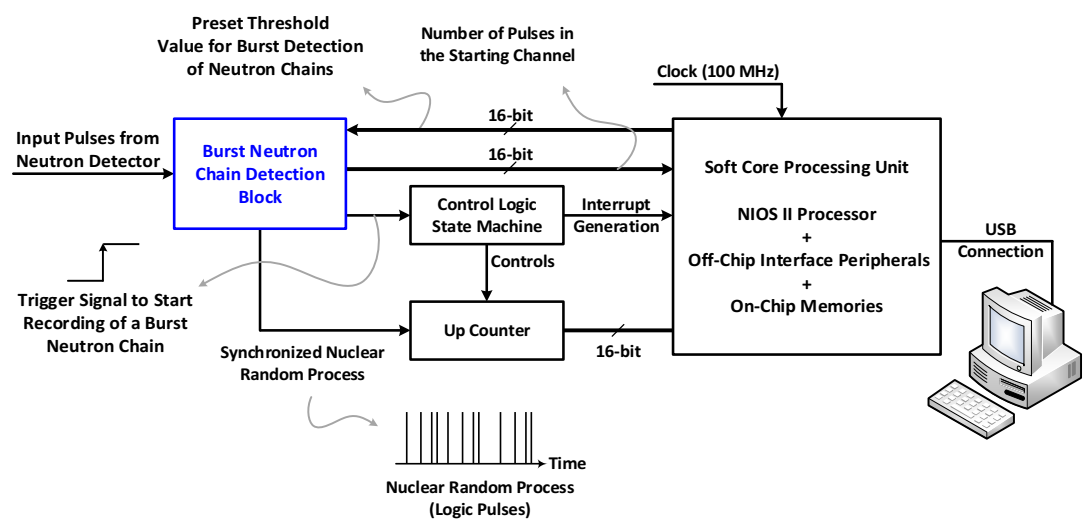

Fig. 2. A general block diagram of the correlation monitor for endogenous pulsed neutron source experiment, the system proposed in the present work.

\section{Esfahan heavy-water zero power reactor (EHWZPR)}

A very useful thermal system for experimental research on neutron physics in heavy-water moderated systems is EHWZPR, a heavy-water moderated nuclear research reactor. In this system, natural metallic uranium fuel elements are arranged in a $20 \mathrm{~cm}$ lattice pitch. The periphery of the core is filled with graphite as a reflector blanket. The present configuration includes 112 fuel elements. The structural materials including clad, top and bottom grid plates 
and reactor tank are made of nuclear-grade aluminium alloy. The cylindrical reactor tank dimensions are around $240 \mathrm{~cm}$ in diameter and $250 \mathrm{~cm}$ in height. A typical thermal power of the reactor is equal to $10 \mathrm{~W}$. It is also possible for the reactor to operate at $100 \mathrm{~W}$ based on ratified experimental procedures approved by the reactor safety committee. The main actuator for reactor control is a precise water level adjusting system.

Two independent and diverse safety systems are designed as the reactor protection system (two safety rods and three dumping moderator valves). As a design basis accident, in the case of loss of electrical power, the safety rods drop into the core by the gravity and within less than three seconds, $80 \mathrm{~cm}$ decrease in water level is guaranteed by three normally open valves of the dumping system. As the moderator is heavy-water, the reactor core's size is big. No fuel depletion calculation is needed, as this is a zero power reactor. Therefore, this is an ideal case study for research purposes on reactor physics. The average prompt neutron lifetime is around $944 \pm 10 \mu \mathrm{s}$ [13] defining the reactor as a markedly thermal system. The present core configuration of the reactor is shown in Fig. 3. Elements of the core are demonstrated graphically. Some general specifications are given in Table 1 [36].

The system can function as a useful facility for research on physical properties of heavywater natural uranium reactors. Also, training of the experimenters and academic teaching, as well as providing a useful tool for verifying and improving theoretical calculations are other major responsibilities of the facility.

The experiment performed in the present work in EHWZPR uses a Helium-3 in-core neutron detector. The position of the detector is located in the physical mid-height of the core. The top view of the location is shown in Fig. 3.

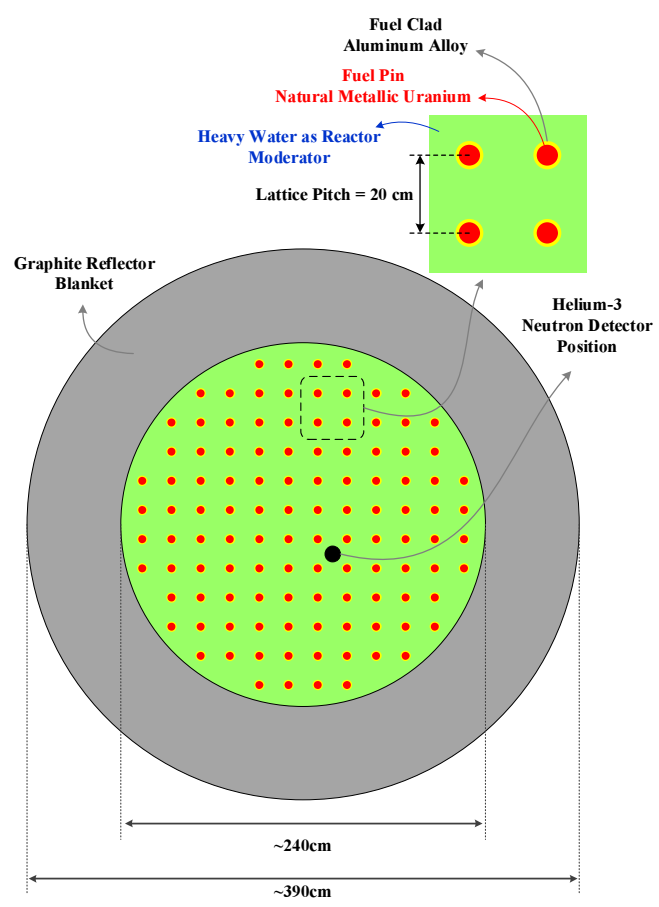

Fig. 3. EHWZPR core configuration; 112 fuel elements are loaded. The location of the in-core neutron detector is also shown. 


\section{Experimental set-up}

Figure 4 shows the experimental set-up employed in this work. To achieve the best timing features, the Helium-3 neutron detector and its preamplifier are assembled in a stainless steel housing. Note that the dead time of the detector used in the present work has been recently studied [32]. The location of the in-core neutron detector is shown in this figure. The preamplifier performs impedance matching as well as amplification of a micro-scale signal provided by the neutron detector. A coaxial cable connects the neutron detector to the amplifier and other nuclear electronic modules located in the laboratory room. Note that when EHWZPR is in operation, the radiation dose level of the reactor hall is increased. Therefore, no operating personnel is allowed to stay in the radiation area. As a result, the remote location of the measuring equipment is obligatory. In the laboratory room, the signal is first amplified and then converted to the logic pulses by a time pick-off module. Three different data acquisition systems are connected in parallel to collect the counting rate of pulses, to perform the Feynman- $\alpha$ analysis, and to measure the correlation of sequences using the endogenous pulsed neutron source data acquisition (DAQ) system. The data collected by each DAQ system are sent to an independent computer located in the laboratory room.

Table 1. A summary of important specifications of EHWZPR.

\begin{tabular}{|c|c|}
\hline Item & Specification \\
\hline Fuel Elements & $\begin{array}{r}\text { Natural Metal Uranium Fuel Rods: } \\
\text { Diameter: } \sim 3.5 \mathrm{~cm} \\
\text { Height: } \sim 200 \mathrm{~cm}\end{array}$ \\
\hline Moderator & Heavy-water \\
\hline Lattice Pitch & $\begin{array}{c}\qquad 20 \mathrm{~cm} \\
\text { (Can be changed in four different lattice pitches) }\end{array}$ \\
\hline Reactor Tank & $\begin{array}{l}\text { Made of Aluminium Alloy: } \\
\qquad \begin{array}{l}\text { Diameter: } \sim 240 \mathrm{~cm} \\
\text { Height: } \sim 250 \mathrm{~cm}\end{array}\end{array}$ \\
\hline Reflector & $\sim 75 \mathrm{~cm}$ Graphite Thickness at the periphery of the core \\
\hline Reactor Thermal Power & $\begin{array}{l}\text { Lower than } 10 \mathrm{~W} \text { (maximum operation time is about half an hour a day) } \\
\text { Lower than } 100 \mathrm{~W} \text { (must be permitted by the reactor safety committee) }\end{array}$ \\
\hline Safety Systems & $\begin{array}{cc}\text { Two diverse and redundant safety systems: } \\
- & \text { Two Safety Rods } \\
- & \text { Emergency Dumping System (Three } \\
& \text { normally open valves) } \\
\end{array}$ \\
\hline Reactor Control & Water level adjustment and two control rods \\
\hline $\begin{array}{c}\ell \\
\text { (Prompt neutron lifetime) }\end{array}$ & $944 \pm 10[\mu \mathrm{s}]$ \\
\hline $\begin{array}{c}\beta_{\text {eff }}[\mathrm{pcm}] \\
\text { (Effective delayed neutron fraction) }\end{array}$ & 665.1 \\
\hline
\end{tabular}

\section{Results and discussion}

In Fig. 5, counting rates of the neutron detector after reactor start-up are shown. During the first $6500 \mathrm{~s}$, heavy water was injected into the core. A multiplication factor of EHWZPR core was controlled by a precise adjustment of the heavy-water level. The injection of the moderator was carried out through six stages in a safe and careful manner; every increase of the water level took about 15 minutes. Water levels are shown in the figure. Around 15 minutes after 
the water level had been adjusted at $1773.4 \mathrm{~mm}$ (to reach the steady state conditions of the system statistics), the data collection for Feynman- $\alpha$ and endogenous pulsed neutron source experiments was started. The data were acquired by the systems in an interval between $7500 \mathrm{~s}$ and $12500 \mathrm{~s}$ (5000 s data collection time). During the experiments, the mean value of neutron counts was about $30000 \mathrm{cps}$. The heavy-water critical level was about $1800 \mathrm{~mm}$. Based on the reactivity value of the moderator $(13.7 \pm 0.41 \mathrm{pcm}$ per millimetre of the heavy-water level of the moderator), an approximate multiplication factor at $1773.4 \mathrm{~mm}$ was estimated to be 0.9963 . During the experiment, the reactor was held in the subcritical state. No external neutron source was employed as background neutrons in the heavy-water moderated system are strongly generated by the photoneutron production.

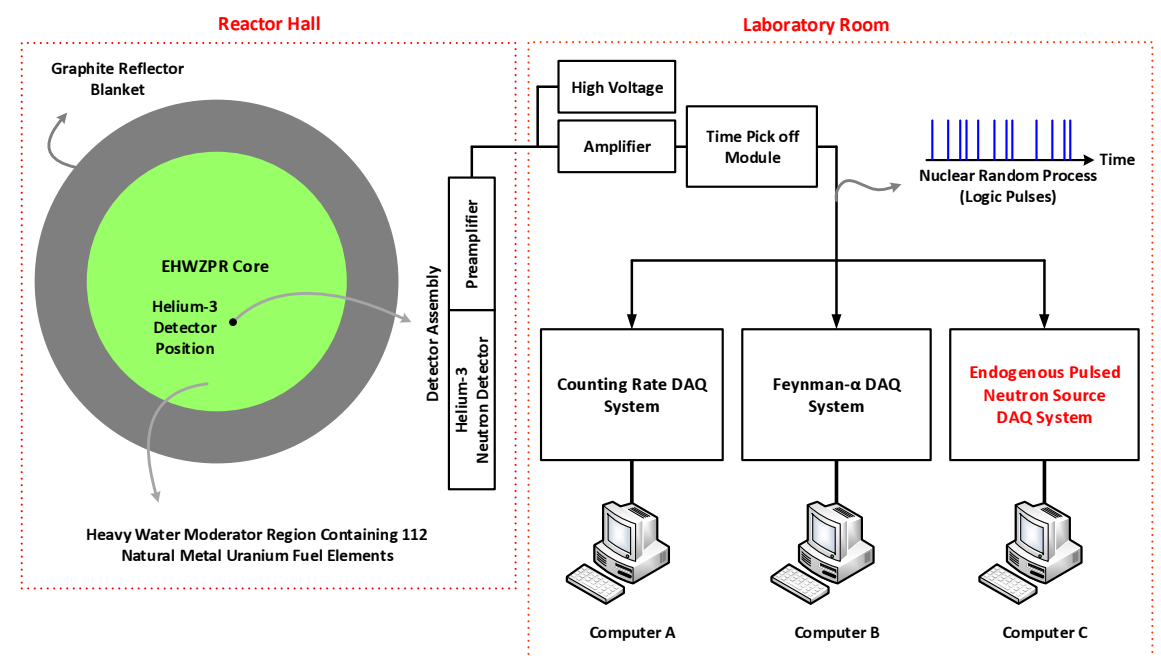

Fig. 4. The set-up of the experiment performed in EHWZPR. A Helium-3 in-core neutron detector is employed.

A DAQ system for measurement of counting rates of the detector is used. Also, two isolated DAQ systems for the Feynman- $\alpha$ and endogenous pulsed neutron source (the system developed in the present work) experiments are specified.

\subsection{Neutron statistics in EHWZPR}

The neutron counts can be considered to have a Gaussian distribution [37]. But in the present case, they have a larger standard deviation than the ones in a non-multiplying medium, when both outputs are of the same mean value. A large value of standard deviation means that there is a high probability for the variable to deviate from its mean value. This large standard deviation becomes noticeable when the reactivity in the multiplying medium approaches the critical state. In the neighbourhood of such data, there are relatively more neutrons that correlate in time with each other.

During the data collection period, one million of $5 \mathrm{~ms}$ samples of integral counts was acquired. A 25 s interval (5000 data points) of the recorded data is shown in Fig. 6 Part-B. The mean count is about 149.8, and its standard deviation is 17.1. In Part-A, a pseudorandom pure Poisson process (the process usually seen in non-multiplying mediums) is shown. Note that in a Poisson process the standard deviation of the counts is equal to the square root of the mean value. A moving average filter is applied to both processes, and the results are illustrated by the red lines. Paroxysmal pulses are clearly seen in Part-B which is labelled as neutron noise fluctuations. Therefore, it is visually confirmed that $\sigma_{Z P R N}$ is greater than $\sigma_{\text {Poisson. }}$. 


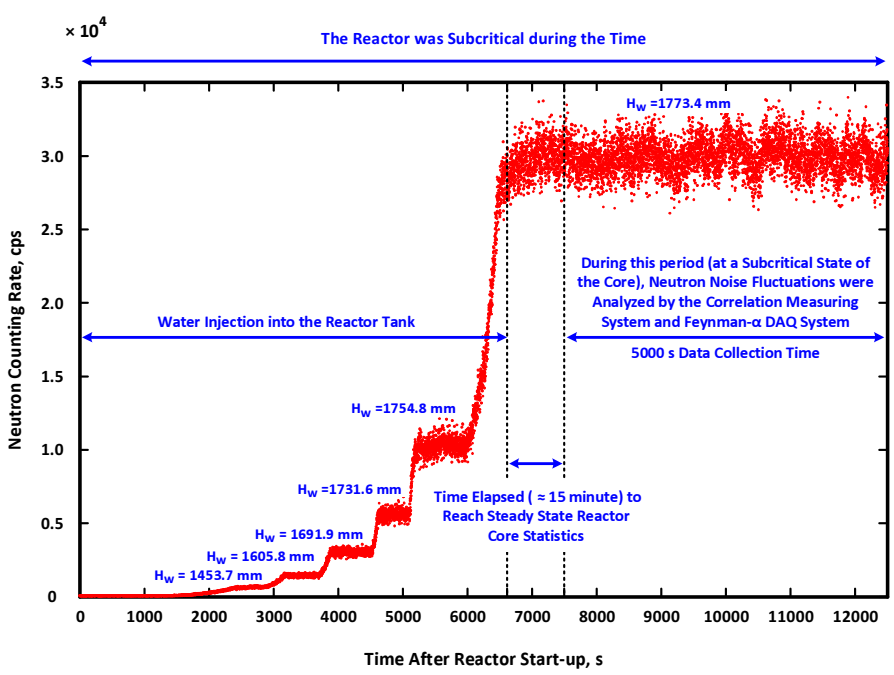

Fig. 5. Neutron counting rates in EHWZPR core after the reactor start-up. From $7500 \mathrm{~s}$ to $12500 \mathrm{~s}$ the data was acquired (around $5000 \mathrm{~s}$ data collection time). During the period, the data on neutron noise fluctuations were collected by the Feynman- $\alpha$ and endogenous pulsed neutron source data acquisition systems.

Figure 7 compares the experimental probability density function (pdf) of the counts with a Gaussian pdf. Accordingly, around the mean value, the probability is more intensive than the experimental pdf. Nonetheless, the distribution measured in EHWZPR shows extra fluctuations around the mean neutron counts. The Gaussian distribution is also fitted to the experimental data. The parameter values are shown in the figure. Now, assume that the Gaussian pdf is the distribution of the background radiation. It is obvious that the experimental pdf is the summation of correlated and background portions. Therefore, the ratio of these functions might be useful to be studied to gain knowledge about a conditional probability of the process. (10) shows the relation between the mentioned $S B R$ parameter and the ratio of the pdfs:

$$
\xi(n)=\frac{P D F_{E}(n)}{P D F_{P}(n)}=\frac{C_{C}+U_{C}}{U_{C}}=\operatorname{SBR}(n)+1 .
$$

In this equation $n$ is a neutron count; $P D F_{E}$ is an experimental pdf of the count and $P D F_{P}$ is a Gaussian pdf. The nominator of (10) is the neutron count affected by the neutron noise fluctuations. Comparing the experimental distribution of neutron statistics in EHWZPR with a pure Poisson distribution as shown in Fig. 7, (with the same average counting rates) a clear deviation from the Poisson distribution occurs due to the correlated chain neutrons. In Fig. 7, variations of the distributions are plotted. A systematic deviation between the experimental data points and the fitted Gaussian curve is obvious. In Fig. 8, the residues of the Gaussian curve fitted to the experimental data are displayed. Although the Gaussian curve is symmetric, the residues have an asymmetric distribution. Regardless of the fluctuations around the mean value $\left(\overline{\mathrm{C}} \pm \sigma_{E x p}\right)$, it is accepted that an observed burst of neutron chains causes a higher abundance on the right-hand side of the curve $\left(C>\left(\overline{\mathrm{C}}+\sigma_{E x p}\right)\right)$, which naturally reduces the probability of abundance of neutron counts on the left-hand side $\left(C<\left(\overline{\mathrm{C}}-\sigma_{E x p}\right)\right)$.

In Fig. 9, on the left axis in a logarithmic scale, $\xi$ is demonstrated. In Region-A, $\xi<1$, that means that the background term is the dominant portion of the process. Therefore, no information can be obtained by the experiments in this region. In regions B and C, the values of $\xi$ are greater than one. But the desired region in the endogenous pulsed neutron source method is Region-C as the system is looking forward to detecting a burst of neutron chains. 
Due to the neutron background, any increase of the threshold level leads to a better selection of the correlated portion of the process (a larger value of $\xi$ ). Therefore, the repetition rate is decreased and a longer collection time is required (refer to the experimental pdf of the counts in EHWZPR, shown in Fig. 7).
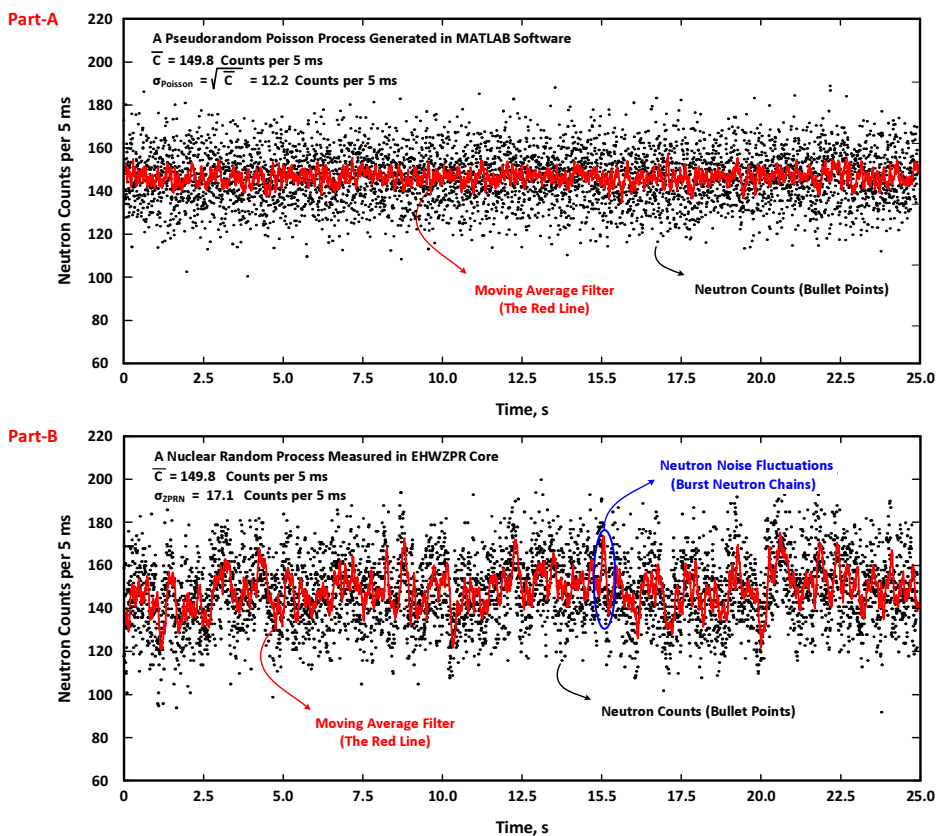

Fig. 6. Part-A: A pseudorandom Poisson process generated in MATLAB software [38].

The mean value of the counts is equal to the mean value of the counts measured in EHWZPR core. Part-B:

Neutron counts within a 5 ms period, measured in EHWZPR. The mean value of the counts is 149.8,

while the standard deviation is 17.1 (noted as $\sigma_{Z P R N}$ in the figure). The measured standard deviation is larger than the standard deviation in the process with a Poisson statistics $\left(\sigma_{Z P R N}>\sigma_{\text {Poisson }}\right)$.

A moving average filteris applied to the data (the red lines) to show neutron noise fluctuations visually.

It is noticeable that there is an inverse relationship between the collection time in the endogenous pulsed neutron source experiment and the parameter $\theta$ value, according to (3) and (4). Combining (4), (5), (6) and (10), and using the fitted Gaussian function to the experimental pdf of the counts ( $\mathrm{PDF}_{\mathrm{E}}$, as shown in Fig. 7), $\theta$ can be expressed as:

$$
\theta=\frac{\xi-1}{T} \int_{\gamma}^{\infty} P D F_{E}(C) d C=\frac{\xi-1}{T} \int_{\gamma}^{\infty} C_{1} \exp \left(-\left(\frac{C-C_{2}}{C_{3}}\right)^{2}\right) d C,
$$

where: $C_{1}$ to $C_{3}$ are constants which are given in Fig. 7 and $C$, a variable of integration, is the number of neutron counts. As the Gaussian fitted curve contains a systematic error shown in Fig. 8, applying the experimental data results in a more accurate definition of the parameter. Using (6), the definition of $\theta$ can be rewritten as:

$$
\theta=\frac{\xi-1}{T} \sum_{i=\gamma}^{\infty} P_{i} .
$$

The values of parameter $\theta$ defined by (12) are calculated at different possible values of $\gamma$, and are plotted in Fig. 10. The optimum selection for $\gamma$ is a range in which $\theta$ is maximized. According to the results shown in Fig. 10 , at $T=5 \mathrm{~ms}$, the best choice for $\gamma$ ranges from 184 to 194. 


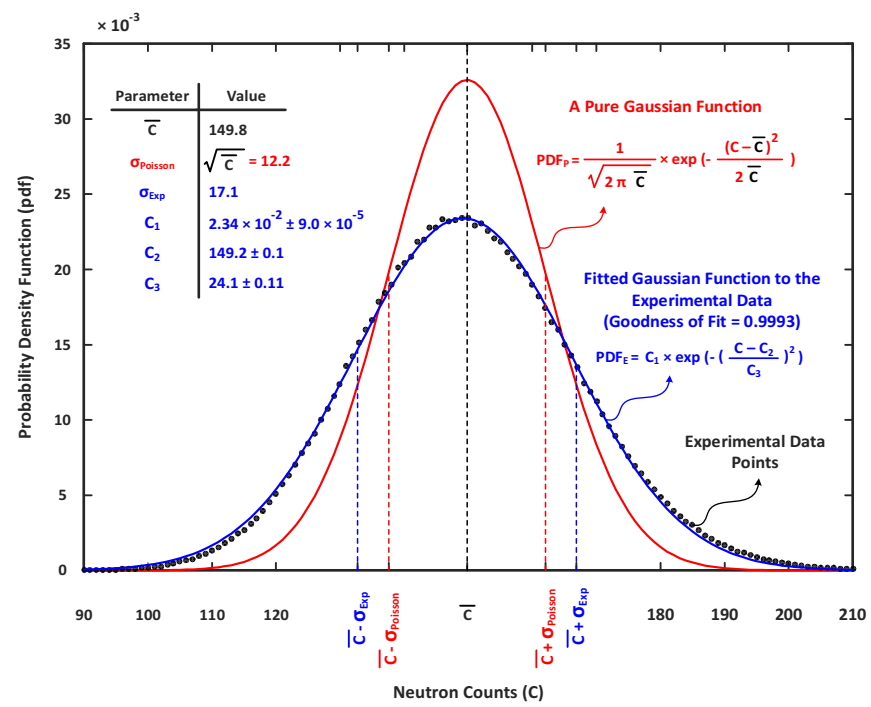

Fig. 7. Comparison of experimental neutron statistics in EHWZPR with a Gaussian pdf of the same mean value.

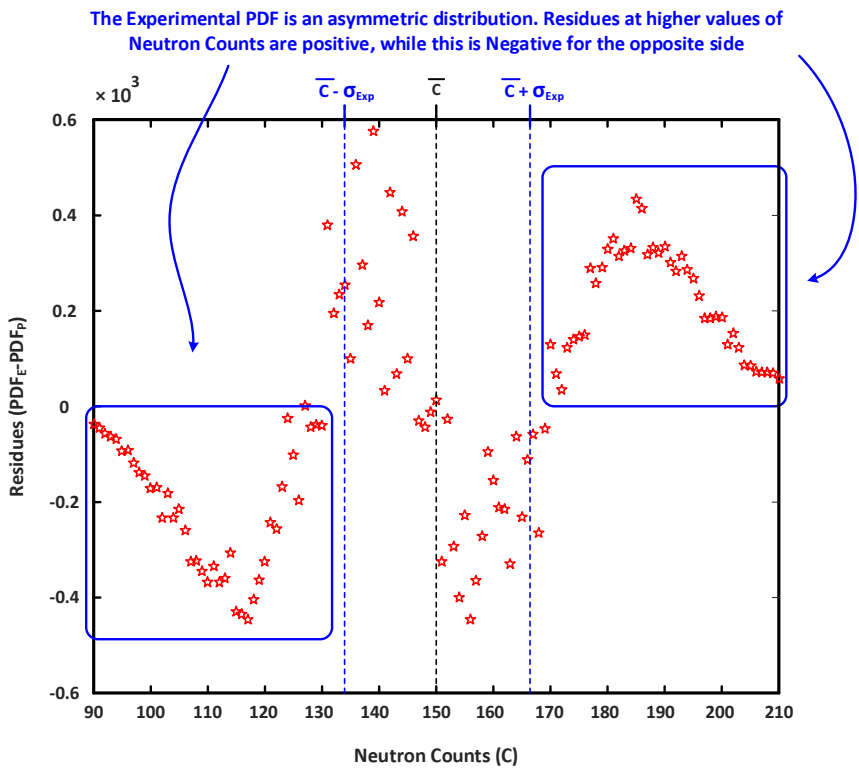

Fig. 8. Residues of the Gaussian function fitted to the experimental data. The results show that the experimental distribution is asymmetric. At higher values of neutron counts $(>170)$, the residues are positive.

On the other side of the distribution, there are negative residues. 


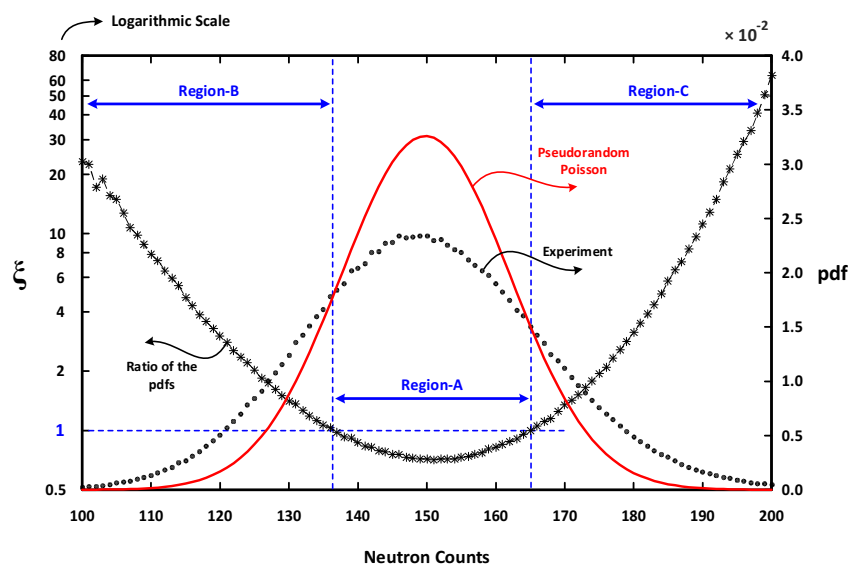

Fig. 9. Values of $\xi$ at different neutron counts. In Region-A, the ratio is smaller than one, therefore the extraction of correlation data is less probable. In Region-B and Region-C, the ratio is larger than one.

Since the endogenous pulsed neutron source experiment is sensitive to positive neutron noise fluctuations, a threshold level must be adjusted in Region-C for neutron burst detection.

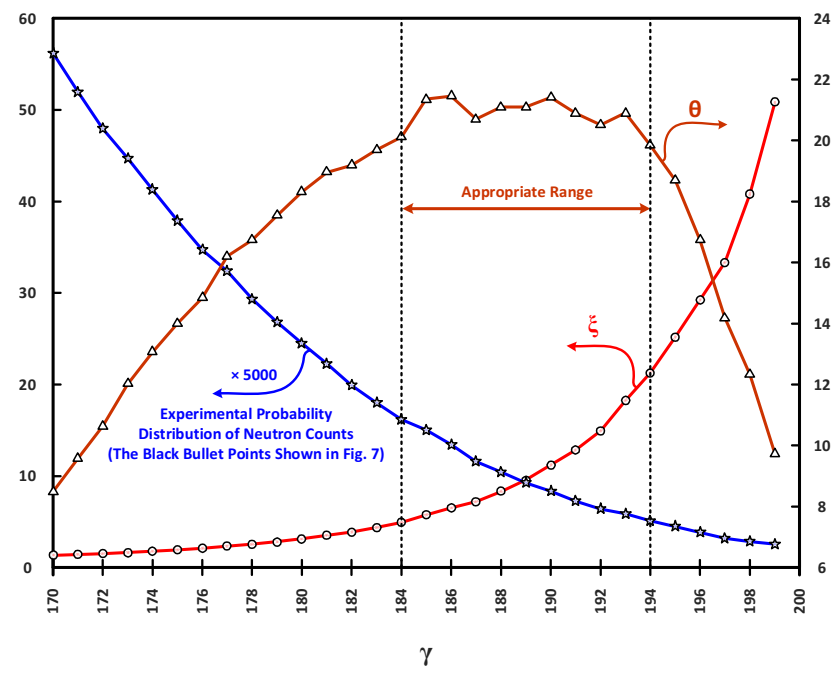

Fig. 10. Values of $\theta$ at different values of $\gamma$. The curves of $\xi$ and $P D F_{E}$ have increasing and decreasing trends, respectively. Therefore, $\theta$ is maximized for some values of $\gamma$.

\subsection{Endogenous pulsed neutron source experiment}

The endogenous pulsed neutron source experiment is carried out in EHWZPR. The EHWZPR core configuration and the experimental set-up are shown in Figs. 3 and 4, respectively. The accuracy of the results is directly related to the number of iterations on the occurrences of neutron bursts applied for the analysis. Therefore, at different numbers of iterations performed by the equipment, the collected data were stored in the memory for postanalysis examinations. In Fig. 11, a few of the results are demonstrated. It is obvious that any increase of the number of iterations improves the statistical accuracy of the results. Note that in EHWZPR the prompt neutron decay constant ranges from $7 \mathrm{~s}^{-1}$ to $20 \mathrm{~s}^{-1}$ based on 
a subcriticality level of the core. That is why the time domain of the total scanning time of the MCS is set to one second. For the most accurate result (item 6 in Fig. 11), the detailed calculation of the neutron decay constant (fitting (1) to the results) is shown in Fig. 12. The lower $\mathrm{x}$-axis represents the time (in seconds) after the triggering pulse, while the upper $\mathrm{x}$ axis - the corresponding MCS channel number. Some selected data at different numbers of iterations are presented in Table 2 in detail. It is clear that an increase of the number of iterations leads to a tighter standard deviation uncertainty of the results, which is confirmed by a better goodness of fit of the curve fittings. The most accurate result for the prompt neutron decay constant is estimated as $8.348 \pm 0.376 \mathrm{~s}^{-1}$. Also, the SBR parameter value of the experiment in EHWZPR is estimated as $(6.04 \pm 0.16) \times 10^{-2}$ (item 14 in Table 2).
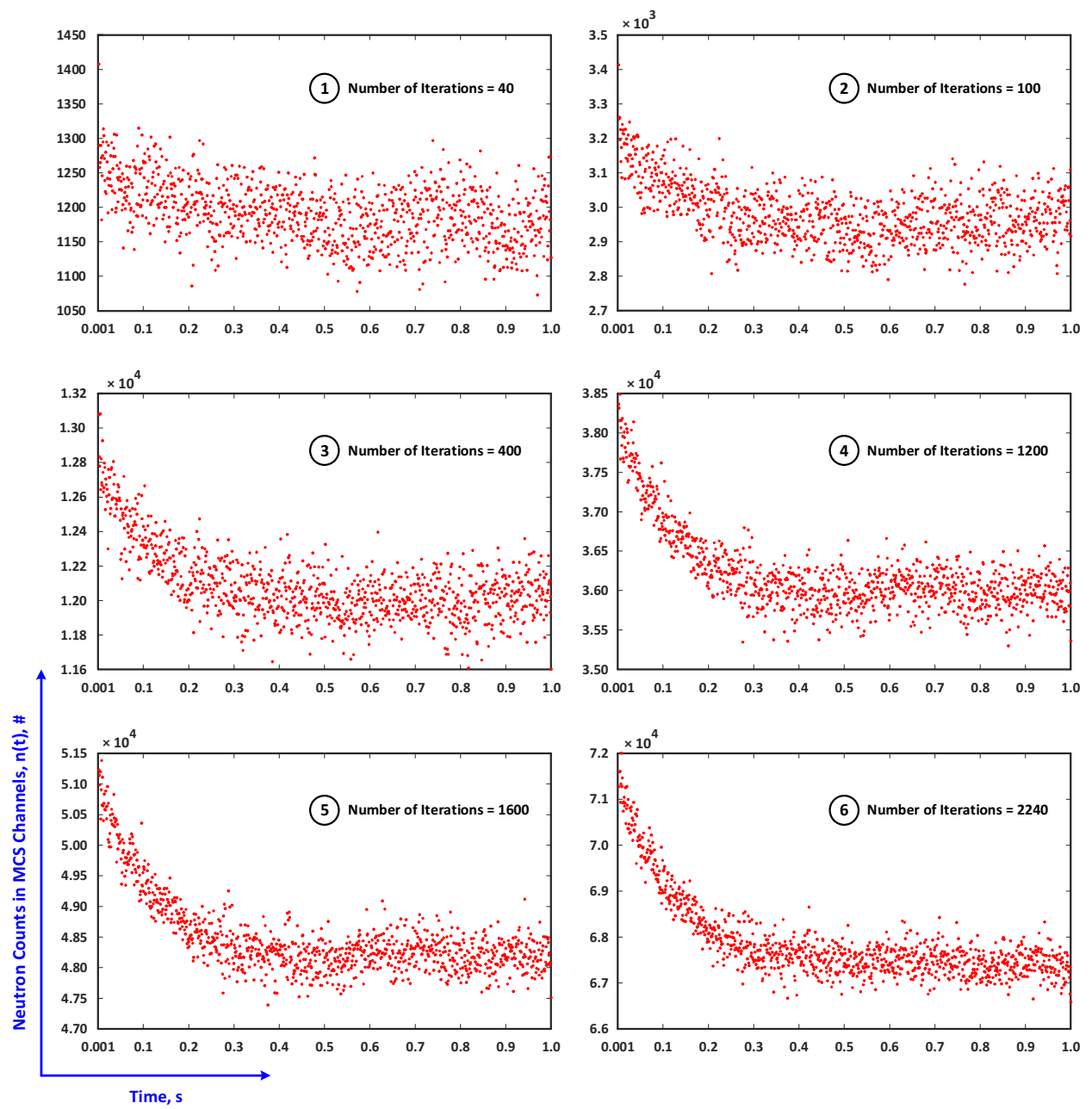

Fig. 11. Results of the endogenous pulsed neutron source experiment at different numbers of iterations on a burst of neutron chains. The item with the least scattered data belongs to the results with the largest number of iterations (item 6). 


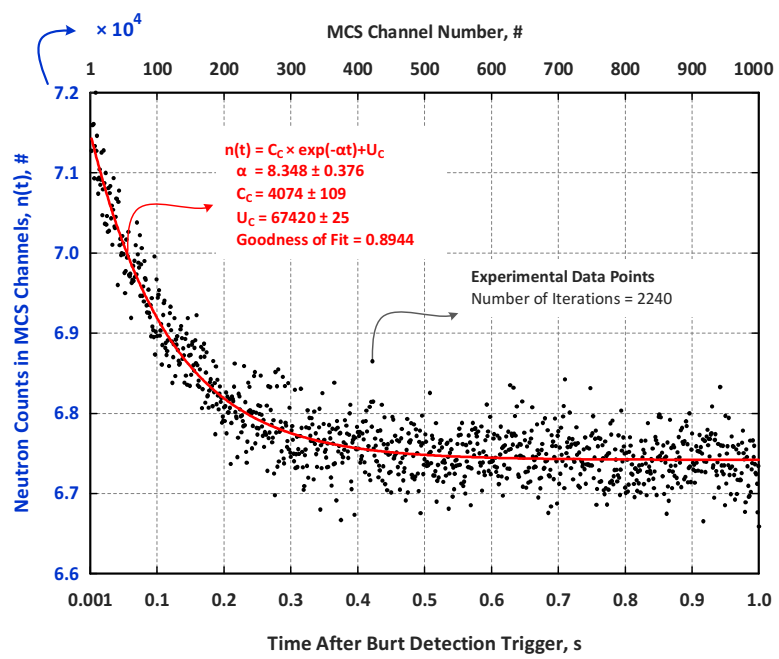

Fig. 12. Results of the endogenous pulsed neutron source experiment in EHWZPR. 2240 iterations on bursts of neutron chains are performed. (1) is fitted to the data and the fitting parameters are shown in the figure. The lower $\mathrm{x}$-axis is time after the triggering pulse, while the upper $\mathrm{x}$-axis is the corresponding MCS channel number.

\subsubsection{Uncertainty analysis of results}

The system was set to store the data collected in MCS channels every 20 iterations performed on the neutron noise fluctuations in the computer memory. Total of 2240 iterations were performed on the results which led to obtaining a $112 \times 2 \times 1000$ data matrix. Cubic interpolations were carried out between the data points to generate finer experimental information. After analysing, the obtained results are presented in Figs. 13 to 15.

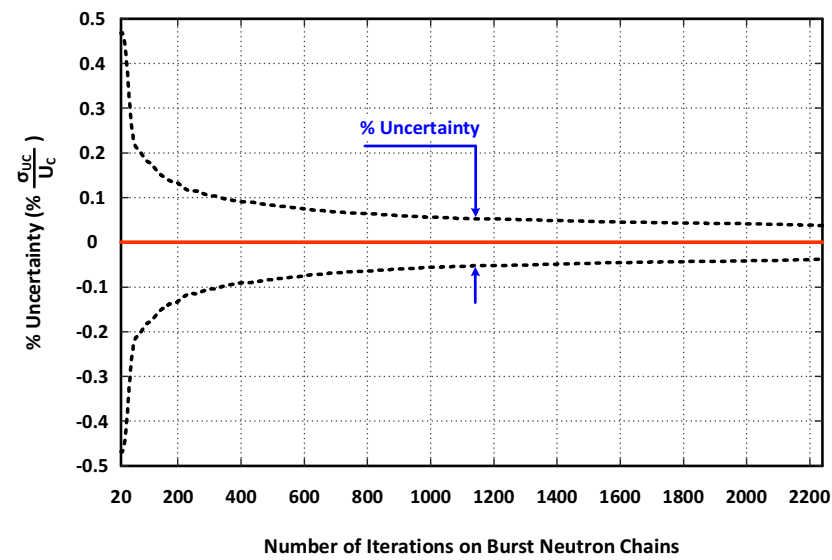

Fig. 13. The experimental uncertainty of uncorrelated counts (UC) at different numbers of iterations on a burst of neutron chains. 


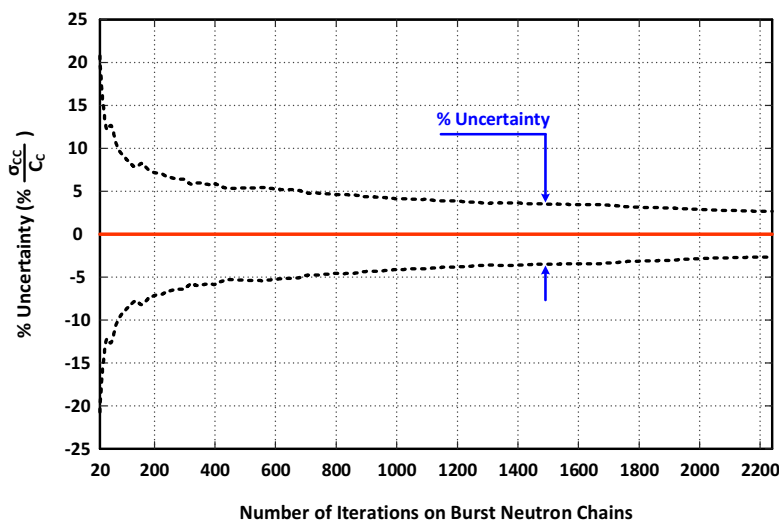

Fig. 14. The experimental uncertainty of correlated counts (CC) at different numbers of iterations on bursts of neutron chains.

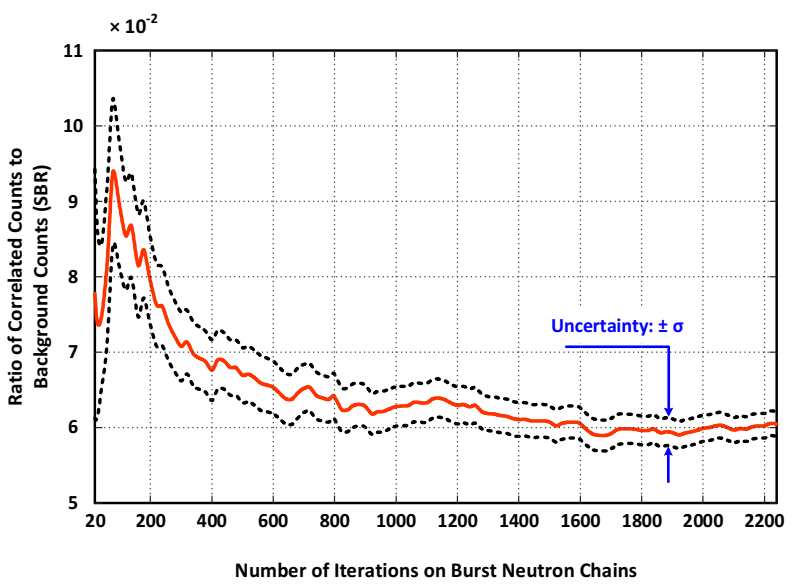

Fig. 15. The ratio of correlated counts and background counts (SBR) measured at different numbers of iterations on bursts of neutron chains.

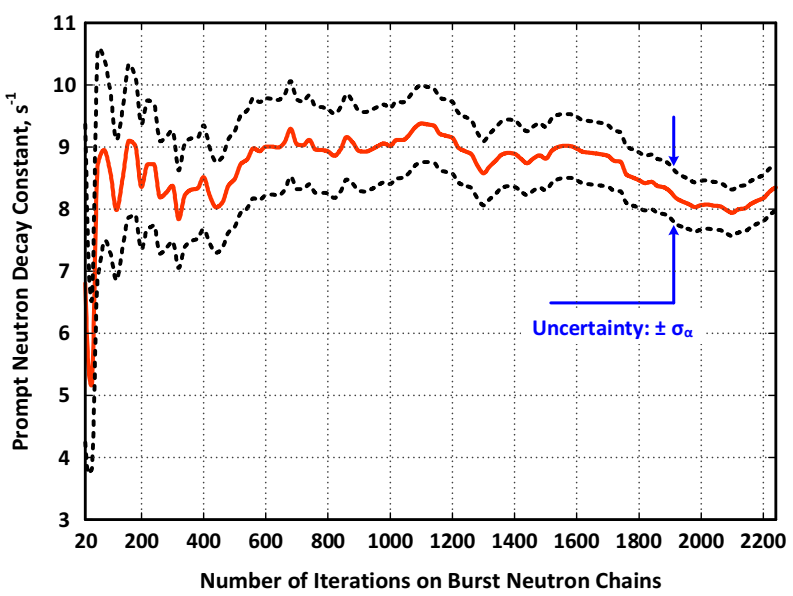

Fig. 16. The experimental results for the prompt neutron decay constant (its mean and its uncertainty boundaries) achieved at different numbers of iterations on the bursts of neutron chains. 
The uncertainty bands, as well as the mean values, are also plotted. The most accurate result is related to the background counts $(\approx \pm 0.03 \%)$ (Fig. 13). The uncertainty of the correlated counts (for 2240 iterations) is $\pm 2.68 \%$. After 600 iterations, the uncertainty was bounded within $\pm 5 \%$, and further iterations led to tighter statistical uncertainties with a short negative slope. The SBR parameter value was also calculated at different numbers of iterations, and the results are shown in Fig. 15. After 1800 iterations, the result was quasi-stable within $\pm 3.2 \%$ of a statistical uncertainty. The error in SBR is mostly originated from the uncertainty of the correlated counts. The most important result, i.e. the prompt neutron decay constant of the core, is shown in Fig. 16. Within up to 1600 iterations, the fluctuating behaviour of the parameter is seen due to the lack of enough collected data on the physical parameter. After that, downward and then upward drifts of the mean value are seen within the statistical uncertainty of the parameter.

Table 2. Parameter values fitted to the experimental data obtained by the endogenous pulsed neutron source experiment for some selected different numbers of iterations on bursts of neutron chains.

\begin{tabular}{|c|c|c|c|c|c|c|}
\hline \multirow[b]{2}{*}{ 希 } & \multirow[b]{2}{*}{ 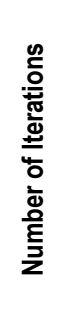 } & \multicolumn{4}{|c|}{ Curve Fitting Parameters } & \multirow[b]{2}{*}{$\begin{array}{c}\text { SBR } \times 10^{2} \\
\text { Dimensionless }\end{array}$} \\
\hline & & $\begin{array}{c}\text { Prompt Neutron } \\
\text { Decay Constant } \\
{\left[\mathrm{s}^{-1}\right]} \\
a \pm{ }^{*} \sigma_{a}\end{array}$ & $\begin{array}{c}\text { Correlated Count } \\
\qquad \#] \\
\mathrm{C}_{C} \pm{ }^{*} \sigma_{\mathrm{Cc}}\end{array}$ & $\begin{array}{c}\text { Uncorrelated } \\
\text { Count } \\
\text { [\#] } \\
U_{c} \pm{ }^{*} \cup_{c}\end{array}$ & $\begin{array}{c}\text { Goodness of } \\
\text { Fit }\end{array}$ & \\
\hline 1 & 40 & $5.160 \pm 1.359$ & $88 \pm 11$ & $1176 \pm 5$ & 0.2355 & $7.48 \pm 0.96$ \\
\hline 2 & 100 & $8.585 \pm 1.294$ & $264 \pm 24$ & $2953 \pm 6$ & 0.4278 & $8.94 \pm 0.82$ \\
\hline 3 & 200 & $8.354 \pm 1.001$ & $476 \pm 34$ & $5989 \pm 8$ & 0.5403 & $7.95 \pm 0.58$ \\
\hline 4 & 300 & $8.378 \pm 0.894$ & $638 \pm 40$ & $9010 \pm 10$ & 0.6005 & $7.08 \pm 0.46$ \\
\hline 5 & 400 & $8.513 \pm 0.835$ & $811 \pm 48$ & $12000 \pm 10$ & 0.6388 & $6.76 \pm 0.40$ \\
\hline 6 & 600 & $9.005 \pm 0.783$ & $1172 \pm 67$ & $17940 \pm 10$ & 0.6898 & $6.53 \pm 0.35$ \\
\hline 7 & 800 & $8.929 \pm 0.676$ & $1536 \pm 70$ & $23970 \pm 15$ & 0.7460 & $6.41 \pm 0.30$ \\
\hline 8 & 1000 & $9.016 \pm 0.612$ & $1879 \pm 28$ & $29940 \pm 15$ & 0.7851 & $6.28 \pm 0.26$ \\
\hline 9 & 1200 & $9.148 \pm 0.578$ & $2264 \pm 87$ & $35980 \pm 15$ & 0.8074 & $6.29 \pm 0.25$ \\
\hline 10 & 1400 & $8.890 \pm 0.533$ & $2571 \pm 93$ & $42120 \pm 20$ & 0.8240 & $6.10 \pm 0.22$ \\
\hline 11 & 1600 & $8.974 \pm 0.510$ & $2915 \pm 100$ & $48180 \pm 20$ & 0.8390 & $6.05 \pm 0.21$ \\
\hline 12 & 1800 & $8.466 \pm 0.446$ & $3232 \pm 102$ & $54230 \pm 25$ & 0.8597 & $5.96 \pm 0.19$ \\
\hline 13 & 2000 & $8.063 \pm 0.394$ & $3607 \pm 104$ & $60210 \pm 25$ & 0.8790 & $5.99 \pm 0.17$ \\
\hline 14 & 2240 & $8.348 \pm 0.376$ & $4074 \pm 109$ & $67420 \pm 25$ & 0.8944 & $6.04 \pm 0.16$ \\
\hline
\end{tabular}

\subsection{Feynman- $\alpha$ experiment}

Within a $5 \mathrm{~ms}$ time window, one million data on neutron noise fluctuations in the EHWZPR core were measured during a $5000 \mathrm{~s}$ period of data collection in the experiment. The bunching method is employed for data analysis. In this method, the time series of data on neutron counts are measured by a MCS with a fundamental gate time channel width. Fig. 17 shows the algorithm of bunching method graphically. Twelve data channels of neutron counts are measured within a gate time of $T$, the basic gate time. The summation of data from every two consecutive channels produces the neutron counts within a gate time of $2 T$. Executing the same 
operation on three consecutive data channels produces the channel data with a $3 T$ gate time width. The same method can be applied for generation of greater values of gate times. The parameter $\mathrm{k}$ is called a combination coefficient, which is an integer number. The postanalysis of the data is performed using the MATLAB software [38]. The bunching method has been widely applied to various research reactors or critical assemblies [13]. The variance to mean ratios of the counts were analysed in time windows of up to one-second widths. The result is shown in Fig. 18; the detailed information estimated by the curve fitting method is also shown in the figure. In Table 3, the Feynman- $\alpha$ result is compared with the data measured by the endogenous pulsed neutron source experiment in EHWZPR. Although the data collection time for both methods is the same, the endogenous pulsed neutron source experiment shows the larger standard deviation error. However, the mean values of the results have a relatively good agreement within the statistical uncertainties. Performing the endogenous pulsed neutron source experiment during a longer data collection period can reduce the uncertainty, as the experiment is a stochastic method of reactor diagnosis.

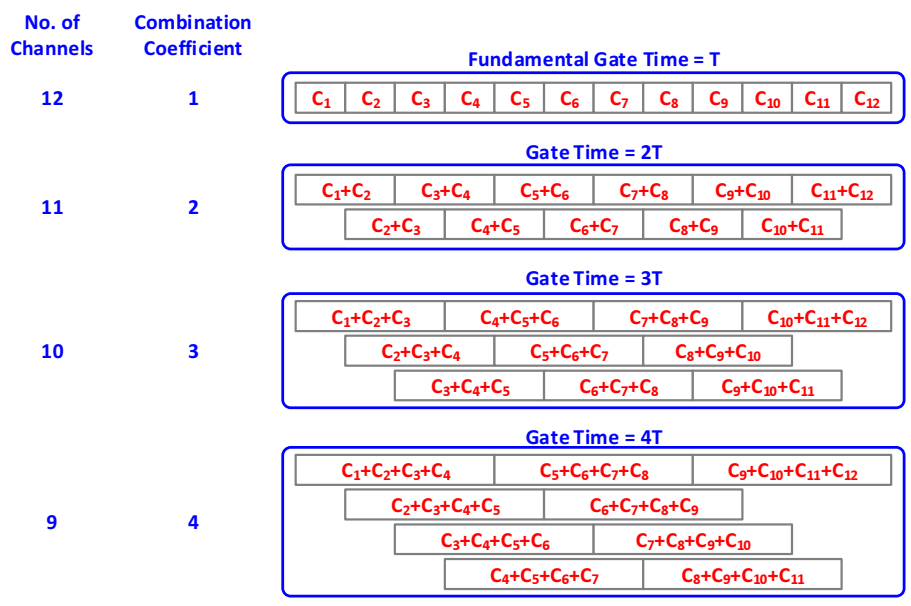

Fig. 17. The concept of bunching method.

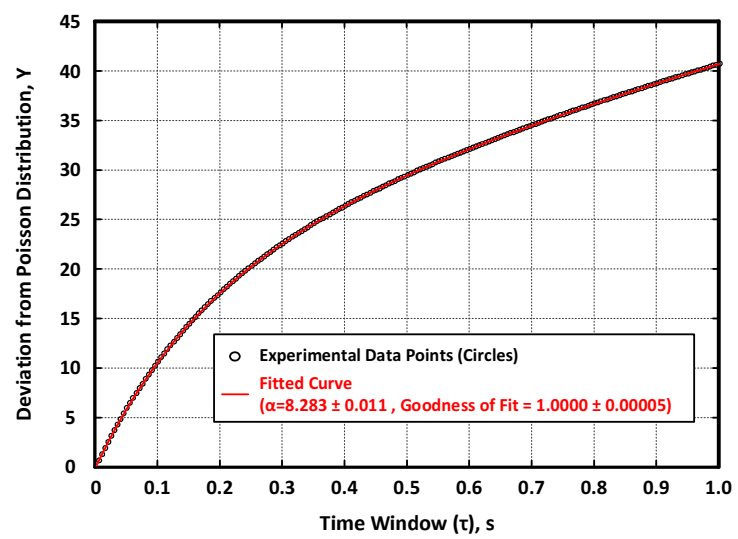

Fig. 18. The Feynman- $\alpha$ results measured in EHWZPR. Fitting parameters are shown in the figure. 


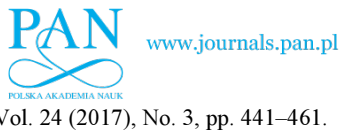

Metrol. Meas. Syst., Vol. 24 (2017), No. 3, pp. 441-461.

Table 3. Comparison of the prompt neutron decay constant measured in EHWZPR using the Feynman- $\alpha$ and endogenous pulsed neutron source experiments.

\begin{tabular}{|c|c|c|c|}
\hline Experimental Method & $\begin{array}{c}\text { Prompt Neutron } \\
\text { Decay Constant } \\
{\left[\mathbf{s}^{-1} \text { ] }\right.}\end{array}$ & Goodness of Fit & Data Collection Parameter \\
\hline Feynman- $\alpha$ & $8.283 \pm 0.011^{\star}$ & $1.0000 \pm 0.00005^{*}$ & $\begin{array}{c}\text { One-million Integral Counts in } 5 \mathrm{~ms} \text { Time } \\
\text { Windows was Collected }\end{array}$ \\
\hline $\begin{array}{c}\text { Endogenous Pulsed } \\
\text { Neutron Source }\end{array}$ & $8.348 \pm 0.376^{*}$ & $0.8944 \pm 0.00005^{\star}$ & $\begin{array}{c}2240 \text { Iterations on Burst Neutron Chains } \\
\text { within } 5000 \text { s were Performed }\end{array}$ \\
\hline
\end{tabular}

${ }^{*}$ Standard Deviation

\section{Conclusion}

The reactor noise experiments have been made as standard methods of reactor diagnosis. A practical alternative method for the Rossi- $\alpha$ experiment in thermal systems is the endogenous pulsed neutron source experiment. The method does not need any special facility. Unlike the pulsed neutron source method, the endogenous pulsed neutron source experiment needs a simple system set-up and reactor operation. The endogenous pulsed neutron source experiment is an efficient method in markedly thermal systems like EHWZPR. In this work, a correlation measuring tool is developed which can detect bursts of neutron chains accurately using a 10-kbit embedded shift register. An MCS module of the system is also included in the design. The system is compact, cost-effective, and uses the state-of-the-art electronic technology. With the aid of the equipment, the endogenous pulsed neutron source experiment was performed in EHWZPR. A prompt neutron decay constant, correlated and uncorrelated counts and SBR parameters were estimated for different numbers of iterations on a burst of neutron chains. A threshold parameter $(\gamma)$ at is crucial for the neutron bursts detection, which is in inverse relation to the collection time required for reaching an acceptable statistical accuracy. An appropriate range of $\gamma$ parameter as well as an uncertainty of the results have been studied. To validate the results, we also performed the Feynman- $\alpha$ experiment. The results obtained by the endogenous pulsed neutron source and Feynman- $\alpha$ experiments have been compared and a good agreement is seen within the statistical uncertainties. The following conclusions are derived:

- An appropriate range of $\gamma$ parameter can be determined using the relation between $\theta$ and $\gamma$. The optimal range of $\gamma$ parameter is obtained for the maximum $\theta$.

- The endogenous pulsed neutron source experiment is a useful and alternative method to be employed in EHWZPR and similar multiplying systems. In such multiplying mediums, the Rossi- $\alpha$ experiment cannot be applied, but the endogenous pulsed neutron source method is applicable.

- The results obtained by the endogenous pulse neutron source experiment are confirmed by the Feynman- $\alpha$ experiment in EHWZPR. Based on the same data collection time (5000 s), the uncertainty of Feynman- $\alpha$ method is tighter than that of the endogenous pulsed neutron source experiment. The endogenous pulsed neutron source method is a stochastic method of reactor analysis. Therefore, if the experiment duration is extended to several hours, better results with a tighter uncertainty might be achieved. For the same data collection time, the results achieved by the Feynman- $\alpha$ method are more accurate than those obtained by the endogenous pulsed neutron source experiment regarding the statistical uncertainty of the results.

- The neutron noise characteristics of the facility was examined with the proposed measuring tool. 
- The system developed in this work is compact, cost-effective, easily adaptable with any special case study, reprogrammable, integrated in a single device, and uses the state-of-theart digital electronic technology.

- A moving time window is designed with a 100 ns time resolution (described in Section 3) using a $10-\mathrm{kbit}$ shift register. Therefore, bursts of neutron chains are detected accurately in time.

\section{References}

[1] Arkani, M., Hassanzadeh, M., Khakshournia, S. (2016). Calculation of six-group importance weighted delayed neutron fractions and prompt neutron lifetime of MTR research reactors based on Monte Carlo method. Progress in Nuclear Energy, (88), 352-363.

[2] Thie, J.A. (1963). Reactor Noise. New York: Rowman and Littlefield Inc.

[3] Uhrig, R.E. (1970). Random Noise Techniques in Nuclear Reactor Systems. New York: The Ronald Press Company.

[4] Williams, M.M.R. (1974). Random Process in Nuclear Reactors. Pergamon Press.

[5] Pázsit, I., Demazière, C. (2010). Noise Techniques in Nuclear Systems. Handbook of Nuclear Engineering. D. Cacuci, Springer US, 1629-1737.

[6] Pázsit, I., Pál, L. (2008). Neutron fluctuations: A treatise on the physics on branching processes. Amsterdam: Elsevier.

[7] Pacilio, N. (1966). Neutron statistics techniques applied to the ROSPO reactor. Proc.s of the Karlsruhe EAES Symposium III, European Atomic Energy Society.

[8] Pacilio, N. (1967-1969). Survey of Advancements in Neutron-Statistics and Reactor-Kinetics Techniques at LFCR. CNEN-RT/FI, (70)29, 1967-1969.

[9] Orndroff, J.D. (1957). Prompt Neutron Periods of Metal Critical Assemblies. Nuclear Science and Engineering, (2), 450-460.

[10] Kitamura, Y., Matoba, M., Misavita, T., Unesaki, H., Shiroya, S. (1999). Reactor Noise Experiments by using Acquisition System for Time Series Data of Pulse Train. Nuclear Science and Technology, (36)8, 653-660.

[11] Arkani, M., Mataji-Kojouri, N. (2016). A Newly Designed Multichannel Scaling System: Validated by Feynman- $\alpha$ Experiment in EHWZPR. Nuclear Engineering and Design, (305) 213-221.

[12] Arkani, M. (2015). A high performance digital time interval spectrometer: an embedded, FPGA-based system with reduced dead time behaviour. Metrol. Meas. Syst., 22(4), 601-619.

[13] Arkani, M. (2015). Measurement of Tehran and Esfahan Research Reactors Kinetic Parameters Using Reactor Noise Diagnostic Methods. Amirkabir University of Technology (formerly called the Tehran Polytechnic), Nuclear Physics Department, Ph.D. Thesis.

[14] Arkani, M., Khalafi, H., Vosoughi, N. (2014). Development of an embedded FPGA-based data acquisition system dedicated to zero power reactor noise experiments. Metrol. Meas. Syst., 22(3), 433-446.

[15] Arkani, M., Khalafi, H., Vosoughi, N., Khakshournia, S. (2015). A FPGA based Time Analyser for Stochastic Methods in Experimental Physics. Instruments and Experimental Techniques, 58(3), 350-358.

[16] Pacilio, N., et al. (1971). A micrologic integrated circuit for monitoring correlation in pulse sequences. Nuclear Instruments and Methods, 92, 13-17.

[17] International Atomic Energy Agency. (1972). Kinetics and Noise Analysis of Zero-Power Reactors: An NPYProject Report, Technical reports series No. 138, Vienna.

[18] Rajeev, K., et al. (2015). Development and testing of neutron pulse time stamping data acquisition system for neutron noise experiment. Nuclear Instruments and Methods in Physics Research Section A: Accelerators, Spectrometers, Detectors and Associated Equipment, 770, 8-13.

[19] Cox, D.R. (1962). Renewal Theory. London: Methuen. 
[20] Smith, W.L. (1957). On renewal theory, counter problems, and quasi-Poisson processes. Mathematical Proceedings of the Cambridge Philosophical Society, 53, 175-193.

[21] Hanus, R., et al. (2014). Velocity measurement of the liquid-solid flow in a vertical pipeline using gammaray absorption and weighted cross-correlation. Flow Measurement and Instrumentation, 40, 58-63.

[22] Zieliński, M., et al. (2009). Accumulated Jitter Measurement of Standard Clock Oscillators. Metrol. Meas. Syst., 16(2), 259-266

[23] Chwaszczewski, S., et al. (1966). Improved Method for Prompt Neutron Period Measurement. Nuclear Science and Engineering, 25(2), 201-202.

[24] Choithramani, S.J. (1981). A comparative study of the shift register and conventional coincidence methods for neutron coincidence counting. Nuclear Instruments and Methods, 180, 189-193.

[25] Murata, Y. (1970). An instrument for analysis of neutron correlation with shift registers. Nuclear Instruments and Methods, 87, 261-272.

[26] Hazama, T. (2003). Practical correction of dead time effect in variance-to-mean ratio measurement. Annals of Nuclear Energy, 30(5), 615-631.

[27] Szappanos, D.G., Por, G., Do, Q.B. (1998). Feynman-alpha measurement in a 100kW research reactor. Progress in Nuclear Energy, 33(4), 439-455.

[28] Kloosterman, J.L., Rugama, Y. (2005). Feynman- $\alpha$ measurements on the fast critical zero-power reactor MASURCA. Progress in Nuclear Energy, 46(2), 111-125.

[29] Spriggs, G.D., Sakurai, T., Okajima, S. (1999). Rossi- $\alpha$ and $\beta$ eff measurements in a fast critical assembly. Progress in Nuclear Energy, 35(2), 169-181.

[30] Szieberth, M., Klujber, G., Kloosterman, J.L., Haas, D.H. (2015). Measurement of multiple $\alpha$-modes at the Delphi subcritical assembly by neutron noise techniques. Annals of Nuclear Energy, 75, 146-157.

[31] Garelis, E., Jr. Russell, J.L. (1963).Theory of pulsed neutron source measurements. Nuclear Science and Engineering, 16(3), 263-270.

[32] Arkani, M., Raisali, G. (2015). Measurement of dead time by time interval distribution method. Nuclear Instruments and Methods in Physics Research, Section A: Accelerators, Spectrometers, Detectors and Associated Equipment, 774, 151-158.

[33] Misawa, T., Shiroya, S., Kanda, K. (1990). Measurement of prompt neutron decay constant and large subcriticality by the Feynman- $\alpha$ method. Nuclear Science and Engineering, 104(1), 53-65.

[34] Keiichi, S. (1979). Source papers in reactor noise. Progress in Nuclear Energy, 3(3), 157-218.

[35] NIOS II Software Developer's Handbook Version 11.1, ALTERA Corporation, 2011.

[36] AEOI, Safety analysis report for Esfahan Heavy Water Zero Power Reactor (EHWZPR), 2013.

[37] Yasuda, H. (1972). Some Remarks on the Endogenous Pulsed Source Technique in Reactor Noise Analysis. Nuclear Science and Technology, 9(8), 501-503.

[38] Mathworks. (2014). MATLAB Reference Guide. The Math Works Inc. 